\title{
Nutritional status, swallowing disorders and respiratory prognosis in adult Duchenne muscular dystrophy patients
}

\author{
Abdallah Fayssoil ${ }^{1}$, Cendrine CHAFFAUT ${ }^{2}$, Helene PRIGENT ${ }^{3}$, Pascal LAFORET ${ }^{3}$, \\ Bernard CLAIR ${ }^{3}$, David ORLIKOWSKI ${ }^{3}$, Adam OGNA ${ }^{3}$, Sylvie CHEVRET ${ }^{2}$, Paris \\ $\mathrm{MENG}^{3}$, Djillali ANNANE ${ }^{3}$, Frédéric LOFASO ${ }^{3}$, and Pascal CRENN ${ }^{3}$ \\ ${ }^{1}$ Hôpital Raymond-Poincare \\ ${ }^{2}$ Hopital Saint-Louis \\ ${ }^{3}$ Hopital Raymond-Poincare
}

October 23, 2020

\begin{abstract}
Malnutrition and swallowing disorders are common in Duchenne muscular dystrophy (DMD) patients. We assessed, in adult DMD with HMV (home mechanical ventilation) and cough assist device, its prevalence and the relationships with respiratory muscle strength and long-term respiratory prognosis. We reviewed the patients $(\mathrm{n}=117$, age 18 to 39 years (median 24)), followed in a reference center, from 2006 to 2015, to obtain clinical baseline, nutritional status, vital capacity (VC), maximal inspiratory pressure (MIP), maximal expiratory pressure (MEP). Median body mass index was low (15.6). Prevalence of malnutrition, swallowing disorders and gastrostomy were respectively 62,34 and 11\%. BMI and serum albumin level were significantly associated with MIP, MEP and VC. The 1/5-years cumulative incidences of respiratory events (pulmonary sepsis and acute respiratory distress) were respectively 20.7/44.5\%. Using univariate analysis, predictive factors for respiratory events were swallowing disorders $(\mathrm{p}=0.001)$, transthyretinemia $(\mathrm{p}=0.034)$, MIP $(\mathrm{p}=0.039)$ and MEP $(\mathrm{p}=0.03)$ but not BMI or albuminemia. Using multivariate analysis, only swallowing disorders remained significantly associated with respiratory events $(\mathrm{OR}=4.2, \mathrm{IC} 95 \%$ 1.31-12.2, $\mathrm{p}=0.01)$. If prevalence of malnutrition and swallowing disorders are high in adult DMD on HMV, and respiratory muscles strength associated with serum albumin level and BMI, respiratory prognosis is mainly related to swallowing disorders.
\end{abstract}

\section{Hosted file}

Final manuscript DMD respi nutri oct 2020.pdf available at https://authorea.com/users/369602/ articles/488427-nutritional-status-swallowing-disorders-and-respiratory-prognosis-inadult-duchenne-muscular-dystrophy-patients 